\title{
Lista preliminar de dípteros de importancia forense en Guayaquil
}

\section{Preliminary List of forensic relevant Diptera in Guayaquil}

Jorman Arbeláez. ${ }^{1}$, Andrea E. Narváez. ${ }^{2}$

Recibido: 10-02-2019 / Revisado: 15-02-2019 /Aceptado: 04-03-2019/ Publicado: 12-03-2019

\begin{abstract}
.
DOI: https://doi.org/10.33262/cienciadigital.v3i1.1.363
\end{abstract}

Forensic entomology uses the presence, abundance and order of arrival of arthropods to a corpse, to provide key evidence regarding criminal investigations. This research represents the first work of entomofauna related to corps in Guayas. We captured and identified adult diptera associated with corpses of guinea pigs (Cavia porcellus) in two locations within Guayaquil city. Van Someren Rydon traps were used to capture flies. 4114 individuals were collected and belong to four families, 18 genera and 29 species. Monte Sinaí locality showed the greater number of species related to guinea pig's corpse, the most dominant species of Diptera observed during the work were Chrysomya albiceps and Musca doméstica, which could serve as indicators for estimation of the Post Mortem Interval, since they were abundant across all decomposition stages. We found species restricted to a specific sampling site, for example, Mesembrinella bellardiana was only found on the Canoa trail at BPCB, while Ophyra aenescens and Atherigona orientalis were found exclusively in Monte Sinaí, such specificity confers them high relevance for forensic investigation regarding cases of corpse transfers.

Keywords: Forensic entomology, Diptera, Guayaquil, Decomposition, Criminalistics.

\section{Resumen.}

La entomología forense utiliza la presencia, abundancia y colonización de artrópodos en un cadáver para brindar evidencia durante una investigación criminal. Esta investigación representa el primer trabajo sobre la evaluación de entomofauna

\footnotetext{
${ }^{1}$ Universidad de Guayaquil, Facultad de Ciencias Naturales, Ecuador. arbelaezjorman@ hotmail.es

${ }^{2}$ Universidad de Guayaquil, Facultad de Ciencias Naturales, Ecuador. aenarvgarc@gmail.com
} 
relacionada a cadáveres en la provincia de Guayas y consistió en la captura e identificación de dípteros asociados a cadáveres de cobayos, Cavia porcellus, en dos localidades dentro de la ciudad de Guayaquil. Se utilizaron trampas Van Someren Rydon modificadas para la captura de moscas y se registró temperatura y pluviosidad en cada colecta. Se colectó 4114 individuos, pertenecientes a cuatro familias, 18 géneros y 29 especies. La localidad Monte Sinaí presentó un mayor número de especies de dipteros asociados al cadáver de cobayo, las especies más dominantes durante el trabajo fueron Chrysomya albiceps y Musca doméstica, las cuáles podrían servir como indicadores en la estimación del IPM. Se encontró especies restringidas a cada localidad, así, por ejemplo, Mesembrinella bellardiana está únicamente en el sendero Canoa, mientras que Ophyra aenescens y Atherigona orientalis se encontraron exclusivamente en Monte Sinaí, lo cual les otorga relevancia para la investigación forense en casos de traslados de cadáveres.

Palabras claves: Entomología forense, Díptera, Guayaquil, Descomposición, Criminalística.

\section{Introducción.}

La materia en descomposición resultante de la muerte de un organismo representa un micro ecosistema sumamente rico y diverso para una variedad de seres con hábitos tróficos y etológicos diferentes (Arnaldos, García y Presa, 2010). Vertebrados e invertebrados carroñeros y hongos saprófitos son atraídos al recurso alimenticio que representan los restos biológicos (Mego, 2017). Durante el proceso de descomposición el cadáver atraviesa una serie de cambios físicos, químicos y biológicos que determinarán el orden de llegada de dichos organismos; así como su diversidad y abundancia (Horenstein, Arnaldos, Rosso y García, 2005).

Para García-Ruilova y Donoso (2015), la entomología forense representa un gran aliado para resolver los problemas judiciales en Ecuador, al brindar información relevante, en función de la presencia de ciertos grupos de artrópodos, sobre casos en los cuáles algunas circunstancias del crimen escapan a los métodos de la criminalística común. Debido a la enorme diversidad geográfica en el Ecuador, se dificulta la generalización de listas de especies de entomofauna asociada a cuerpos en descomposición. Por esta razón, los estudios en el país deben centrarse alrededor de las ciudades estratégicas en cada una de las regiones, dando prioridad a los sitios donde los cadáveres suelen ser arrojados y trabajando en coordinación con la Policía Nacional (Keil, 2015).

\section{Fundamento Teórico}

La entomología forense es una rama de las ciencias criminalísticas que utiliza la presencia, abundancia y orden de colonización de insectos y demás artrópodos en un cadáver (Villanueva y Seclen, 2016). La efectividad de la entomología forense, para la resolución de 
crímenes en países faunísticamente diversos, ha sido ampliamente reportada y permite determinar factores generales como intervalos post-mortem y traslados (Bugelli et al., 2015). La identificación correcta de las especies, el conocimiento de sus ciclos de vida y de su orden de llegada al cadáver, brindan las herramientas necesarias para conocer el Intervalo Post Mortem (IPM), la causa y el lugar de la muerte (Beltrán y Navarro, 2012; Peceros, 2011) y ha permitido también resolver casos más específicos como la toxicología en envenenamientos (Byrd y Castner, 2009). La entomología forense ha demostrado ser la mejor técnica para conocer el IPM, al ser mucho más efectiva que las autopsias, las confesiones y los testimonios de testigos (Gruner, 2004).

Cuando una persona muere, su cadáver se transforma en un recurso trófico. La llegada de los insectos al cuerpo en descomposición no es simultánea, sino que se presenta en oleadas denominadas "cuadrillas". Las cuadrillas dependen de factores climáticos y biogeográficos; cada una de ellas se encuentra caracterizada por la presencia de órdenes particulares de insectos (Centeno, 2016). El orden de llegada de las cuadrillas de insectos es, hasta cierto punto, predecible y guarda relación con los hábitos alimenticios, etología y composición de la comunidad de insectos de cada región, los cuáles obtienen diversos beneficios del cadáver y a su vez lo modifican (Ramos-Pastrana, Virgüez-Díaz y Wolff, 2018). Zanetti, Visciarelli y Centeno (2015) indican que la capacidad de detectar cambios químicos asociados a la materia en descomposición que poseen ciertos insectos hace que su presencia no sea casual y que pueda servir como un indicador para la estimación del IPM.

El establecimiento de catálogos de especies con importancia forense es un procedimiento necesario para la correcta aplicación de esta técnica (Salazar y Donoso, 2015). Sin embargo, la diversidad de insectos necrófagos, sus hábitos alimenticios, patrones de distribución espacial son aún poco explorados. Así también, se desconoce la influencia de las condiciones climáticas en la incidencia de insectos en un cuerpo, es por esto que este trabajo busca brindar la primera lista preliminar de dípteros de importancia forense asociados a cadáveres de cobayos (Cavia porcellus) en dos sectores de la ciudad de Guayaquil y analizar su importancia para la aplicación de la entomología forense.

\section{Metodologia.}

Este trabajo se llevó a cabo en dos sitios de la ciudad de Guayaquil. La ciudad posee un clima cálido-húmedo, con dos estaciones bien diferenciadas: la época lluviosa que abarca los meses de diciembre hasta abril y la época seca considerada entre mayo y noviembre (Temperatura promedio $32^{\circ}$, Humedad promedio $80 \%$ ) (Johansson, Yahia, Arroyo y Bengs, 2018). La primera localidad es una residencia situada en el sector de la cooperativa Monte Sinaí, parroquia Tarqui, (-02¹0'00.0663" S, $\left.-080^{\circ} 01^{\prime} 21.2695 " E\right)$ al noroeste de la ciudad de Guayaquil. Este sitio se eligió con la intención de simular los asesinatos que ocurren en el perímetro urbano de la ciudad. La segunda localidad está en el Bosque Protector Cerro Blanco (BPCB), parroquia Chongón, (-0207'11.0480" S, -07958'42.6374" E). Este punto 
permitió detectar la descomposición de los cadáveres y la composición de la comunidad de dípteros en un área de bosque. Los muestreos se realizaron en el sendero Canoa, el más corto de todo el bosque protector con 1177 metros de longitud (Illescas y Perez, 2009). Esta zona cuenta con árboles de 25 metros de altura y un estrato herbáceo, es considerada una zona intervenida y de fácil acceso (Alava, 2015) simulando los sectores donde usualmente los cadáveres son abandonados.

La trampa Van Someren Rydon con la que se capturaron los dípteros adultos, consiste en un cilindro fabricado con organza de aproximadamente $80 \mathrm{~cm}$ de largo y $10 \mathrm{~cm}$ de radio, sostenido con alambres en ambos extremos, cerrado en la parte superior y termina en un embudo de $5 \mathrm{~cm}$ de radio en la base (Lucci Freitas et al., 2014). El diseño que se usó en esta investigación tomó como referencia el utilizado por Amat et al. (2013) y fue modificado a partir del modelo cedido por el Departamento de Entomología de la Pontificia Universidad Católica del Ecuador.

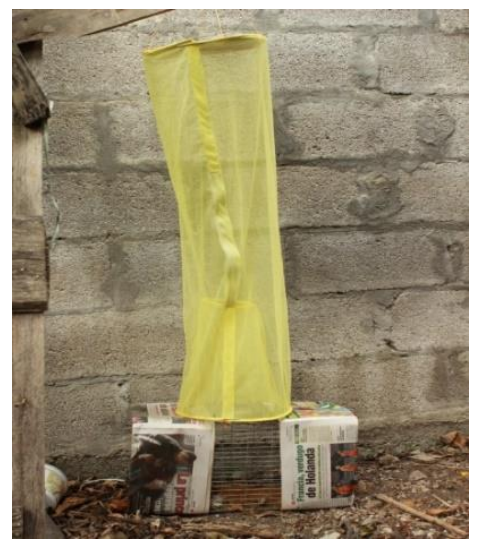

Figura 1. Jaula conteniendo el cadáver de Cavia porcellus y tramapa Van Someren Rydon para la captura de dipteros adultos.

Los muestreos estandarizados permitieron capturar e identificar los dípteros de hábitos necrófagos. Se utilizaron diez cobayos (Cavia porcellus) de tamaño mediano. El tiempo de trampeo duró entre 13 y 17 días en cada localidad debido a las variaciones existentes en la descomposición de cada cobayo. Los animales fueron sacrificados por medio de trauma en la región cervical. Los cuerpos de dichos cobayos fueron colocados en jaulas metálicas fijadas al suelo para evitar que sean perturbados por vertebrados; posteriormente, se colocaron sobre las jaulas las trampas Van Someren-Rydon.

La identificación taxonómica fue realizada en el laboratorio de microscopía de la facultad de Ciencias Naturales, utilizando las claves dicotómicas de Domínguez y Aballay (2014), Buenaventura, Camacho, García, y Wolff (2009), Amat, Velez, y Wolff (2008) y Carvalho y Mello-Patiu (2008). Se utilizó un estereomicroscopio (Carl Zeiss modelo DV4). Se consideró el nivel taxonómico inferior posible: especie o género cuando fue posible la identificación. Para conocer el aporte de cada especie al número total de individuos de calculó 
el índice de importancia relativa basado en el procedimiento establecido por Hart, Calver y Dickman (2002).

\section{Resultados.}

Se recolectó un total de 4114 individuos divididos en cuatro familias, 18 géneros y 29 especies. El sendero Canoa (BPCB) presentó el mayor número de especies $(\mathrm{N}=20)$ a diferencia de Monte Sinaí que presentó $N=19$. Se encontró una mayor cantidad de individuos en Monte Sinaí (2272) en comparación con Sendero Canoa (1842). La familia más abundante fue Calliphoridae con 2491 individuos colectados a lo largo de todo el monitoreo (60.5\%), seguido de Muscidae con 821 (19.9\%) y Sarcophagidae con 682 (16.5\%). La familia menos abundante fue Fanniidae (2.06\%) con 85 individuos.

Tabla 1. Lista de especies y géneros encontrados en las dos localidades muestreadas

\begin{tabular}{|c|c|c|}
\hline Especie & Sendero Canoa & Monte Sinaí \\
\hline Chrysomya albiceps & $x$ & $x$ \\
\hline Chrysomya megacephala & $x$ & $x$ \\
\hline Lucilia sericata & $x$ & $x$ \\
\hline Lucilia cuprina & $x$ & $x$ \\
\hline Lucilia eximia & & $x$ \\
\hline Mesembrinella bellardiana & $x$ & \\
\hline Cochliomyia macellaria & $x$ & $x$ \\
\hline Paralucilia fulvinota & $x$ & \\
\hline Compsomyiops furvicrura & & $x$ \\
\hline Lucilia sp1 & $x$ & \\
\hline Lucilia sp2 & $x$ & \\
\hline Lucilia sp3 & $x$ & \\
\hline Calliphorinae sp & $x$ & \\
\hline Chrysomya sp1 & $x$ & \\
\hline Peckia lambens & $x$ & $x$ \\
\hline Peckia sp & $x$ & $x$ \\
\hline Sarcodexia sp & $x$ & $x$ \\
\hline Titanogrypa sp & $x$ & $x$ \\
\hline Sarcophaga sp & & $x$ \\
\hline Morelia sp & & $x$ \\
\hline Helicobia sp & & $x$ \\
\hline Sarcofahrtiopsis sp & $x$ & \\
\hline Tricharaea sp & $x$ & \\
\hline Sarcophagidae sp & & $x$ \\
\hline Musca domestica & $x$ & $x$ \\
\hline Ophyra aenescens & & $x$ \\
\hline Atherigona orientalis & & $x$ \\
\hline
\end{tabular}


El IRI demostró que las especies mayormente capturadas fueron Chrysomya albiceps $(25,9$ $\%)$ seguida por Musca domestica (13,8 \%) y Lucilia sericata $(13,07 \%)$, mientras que las menos abundantes fueron Compsomyiops furvicrura $(0,048 \%)$.

Tabla 2. Índice de importancia relativa de cada especie reportada caracterizado en función del número de individuos colectados durante cuatro muestreos.

\begin{tabular}{ll}
\hline Especie & IR \\
\hline Chrysomya albiceps & 25,86 \\
Chrysomya & 2,26 \\
megacephala & \\
Lucilia sericata & 13,08 \\
Lucilia cuprina & 11,79 \\
Lucilia eximia & 0,46 \\
Mesembrinella & 3,06 \\
bellardiana & \\
Cochliomyia macellaria & 3,14 \\
Paralucilia fulvinota & 0,12 \\
Compsomyiops & 0,05 \\
furvicrura & \\
Lucilia sp1 & 0,19 \\
Lucilia sp2 & 0,19 \\
Lucilia sp3 & 0,19 \\
Calliphorinae sp & 0,05 \\
Chrysomya sp1 & 0,10 \\
Peckia lambens & 4,62 \\
Peckia sp & 2,84 \\
Sarcodexia sp & 4,08 \\
Titanogrypa sp & 4,04 \\
Sarcophaga sp & 0,07 \\
Morelia sp & 0,05 \\
Helicobia sp & 0,07 \\
Sarcofahrtiopsis sp & 0,12 \\
Tricharaea sp & 0,12 \\
Sarcophagidae sp & 0,80 \\
Musca domestica & 13,81 \\
Ophyra aenescens & 2,19 \\
Atherigona orientalis & 4,50 \\
Muscidae sp & 0,07 \\
Fanniia sp & 2,07 \\
\hline &
\end{tabular}


Las 10 especies dominantes encontradas en este trabajo fueron: Chrysomya albiceps, Chrysomya megacephala, Lucilia sericata, Lucilia cuprina, Cochliomyia macellaria, Peckia lambens, Peckia sp, Sarcodexia sp, Titanogrypa sp y Musca doméstica. La especie Mesembrinella bellardiana es reportada únicamente en el sendero. Compsomyiops furvicrura es reportada solo para el estado fresco en Monte Sinaí, mientras que Paralucilia fulvinota fue encontrada solo en el sendero Canoa. Los géneros Sarcodexia, Tytanogrypa y Peckia pertenecientes a la familia Sarcophagidae se encontraron en todos los estados en las dos localidades mientras que Morelia y Helicobia fueron únicamente reportados en Monte Sinaí y Sarcofahrtiopsis y Tricharaea se encontraron solo en Sendero Canoa. Musca domestica fue reportada en las dos localidades mientras que Ophyra aenescens y Atherigona orientalis, también miembros de la familia Muscidae fueron reportadas únicamente en Monte Sinaí. La familia Fanniidae fue reportada en Monte Sinaí.

\section{Discusión}

Este trabajo representa la primera aproximación al estudio de la entomología forense en la provincia de Guayas. El cobayo (Cavia porcellus) es un modelo biológico útil para investigaciones que tengan como finalidad el establecimiento de catálogos y determinación de abundancia y diversidad-

Las tres familias predominantes en las dos localidades fueron Calliphoridae, Muscidae y Sarcophagidae, mientras que hubo ocasionales colectas de miembros de Fanniidae.

Chrysomya albiceps y Musca domestica fueron las dos especies más abundantes en cada estado de descomposición en ambos sitios por lo que representan especies de relevancia para estudios forenses que involucren la estimación del IPM mediante el análisis del desarrollo larvario. Mesembrinella bellardiana fue reportada solamente en el sendero Canoa mientras que Ophyra aenescens y Atherigona orientalis se encontraron solo en Monte Sinaí; estas tres especies son relevantes en caso de traslados del cuerpo, esto en casos donde el sitio de descubrimiento del cadáver no sea el mismo en el cual se cometió el crimen. Es importante mencionar que al igual que en el trabajo de Ramos et al. (2014), en esta investigación Ophyra aenescens fue reportada dentro de una vivienda (Monte Sinaí), mientras que Mesembrinella bellardiana fue reportada solamente en el sendero Canoa de BPCB, reflejando un resultado similar al obtenido por Vasconcelos y Araujo (2012), quienes también encontraron esta especie solo en ambientes forestales.

Entre algunas de las conductas observadas está el carácter predador de la familia Sarcophagidae, en especial del género Peckia. Este fenómeno fue reportado por Beltrán y Navarro (2012), en el cuál los miembros de esta familia se alimentan de larvas de Califóridos y demás dípteros, disminuyendo en gran cantidad el número de individuos que alcanzan el estado de pupa. Otro fenómeno que pudo ser observado durante esta investigación, especialmente en la localidad de sendero Canoa, fue un enorme número de Hymenópteros (en especial hormigas) que fueron atraídas al cadáver una vez alcanzado el estado de 
hinchado. Estos insectos se alimentaron de larvas, restos del cadáver e incluso de dípteros adultos que quedaban atrapados durante el momento de la ovoposición o de la alimentación.

\section{Conclusiones.}

- La importancia de este primer acercamiento a la fauna de interés forense dentro de la provincia de Guayas se refleja en el abanico de oportunidades que abre, con miras a la futura incorporación de la entomología forense en investigaciones criminalísticas en casos de homicidios y negligencias. Sin embargo, las dificultades en la identificación taxonómica y la toma de datos ambientales denotan el estado inmaduro en el cual se encuentran este tipo de investigaciones en el Ecuador, por lo que se sugiere expandir la escala temporal y espacial de estos trabajos mediante convenios que permitan replicarlos en diversas partes del país en distintos ecosistemas y bajo distintas condiciones climáticas.

\section{Referencias bibliográficas.}

Aballay, H., Murua, F., Acosta, C., y Centeno, D. (2012). Succession of Carrion Fauna in the Arid Region of San Juan Province, Argentina and Its Forensic Relevance. Neotropical Entomology, 41(1), 2731.

Alava, L. (2015). Diversidad y abundancia de la quiropterofauna en el Bosque Protector Cerro Blanco como indicador de su estado de conservacion (Tesis de pregrado). Universidad de Guayaquil, Ecuador.

Amat, E., Ramirez Mora, M., y Buenaventura, E. (2013). Variación Temporal De La Abundancia en familias de moscas carroñeras (Diptera, Calyptratae) en un valle andino antropizado de Colombia. Acta Zoológica Mexicana, 29(3), 463-472.

Amat, E., Velez, M., y Wolff, M. (2008). Illustrated key for identification to genera and species of blowflies (Diptera: Calliphoridae) of Colombia. Caldasia, 30(1), 231-244.

Arnaldos, M., García, M., y Presa, J. (2010). Sucesión faunística sarcosaprófaga curso 2010-11 (Material de aula). En Master Universitario en Ciencias Forenses. Universidad de Murcia, España.

Beltrán, A., y Navarro, V. (2012). Sucesión de insectos en cadáveres de ratas Wistar (Muridae: Rattusnorvegicus) (Berkenhout, 1769) en Bosque húmedo Premontano (Ibagué - Colombia). Revista Tumbaga, 6, 93-105.

Buenaventura, E., Camacho, G., García, A., y Wolff, M. (2009). Sarcophagidae (Diptera) de importancia forense en Colombia: claves taxonómicas, notas sobre su biología y distribución. Revista Colombiana de Entomología, 35(2), 189-196.

Bugelli, V., Forni, D., Bassi, L., Di Paolo, M., Marra, D., Lenzi, S., ... Vanin, S. (2015). Forensic Entomology and the Estimation of the Minimum Time Since Death in Indoor Cases. Journal of Forensic Sciences, 60(2), 525-531. doi:10.1111/1556-4029.12647

Byrd, J. H., y Castner, J. L. (2009). Forensic entomology : the utility of arthropods in legal investigations. Florida, USA: CRC Press. 
Carvalho, B de., y Mello-Patiu, C de. (2008). Key to the adults of the most common forensic species of Diptera in South America. Revista Brasileira de Entomologia, 52(3), 390-406. doi: 10.1590/S0085-56262008000300012.

Centeno, C. (2016). Entomología forense: dípteros de interés criminalístico en el fundo la esperanza, sector La Yaguara, Municipio Libertador del estado Carabobo (Tesis de maestría). Universidad de Carabobo, Venezuela.

García-Ruilova, A., y Donoso, D. A. (2015). Casos Sin Resolver y La Entomología Forense en Ecuador. Revista Ecuatorianade Medicina Y Ciencias Biológicas, 36(1), 59-63.

Gruner, S. (2004). The forensically important Calliphoridae (insecta: diptera) of pig carrion in rural north-central florida. Biophysical Journal. University of Florida.

Hart, R., Calver, M., y Dickman, C. (2002). The index of relative importance: an alternative approach to reducing bias in descriptive studies of animal diets. Wildlife Research, 29, 415-421. doi:10.1071/WR02009.

Horenstein, M., Arnaldos, M., Rosso, B., y García, M. (2005). Estudio preliminar de la comunidad sarcosaprófaga en Córdoba (Argentina): aplicación a la entomología forense. Anales de Biología, 27, 191-201.

Illescas, L., y Perez, I. (2009). Evaluacion del uso turistico y recreativo del Bosque Protector Cerro Blanco (Tesis de pregado). Escuela Superior Politécnica del Litoral, Guayaquil, Ecuador.

Johansson, E., Yahia, M. W., Arroyo, I., \& Bengs, C. (2018). Outdoor thermal comfort in public space in warm-humid Guayaquil, Ecuador. International Journal of Biometeorology, 62(3), 387-399. doi: 10.1007/s00484-017-1329-x.

Keil, C. (2015). Research needs for forensic entomology in Ecuador. Revista Ecuatoriana de Medicina y Ciencias Biológicas, 1(36), 71-78.

Lucci Freitas, A., Agra Iserhard, C., Pereira Santos, J., Oliveira CarreiraI, J., Bandini Ribeiro, D., Alves Melo, D. H., ... Uehara-prado, M. (2014). Studies with butterfly bait traps: an overview. Revista Colombiana de Entomología, 40(2), 203-212.

Mego, G. (2016). Descomposición Cadavérica y Determinación del Intervalo Post Mortem. Skopein: La Justicia en manos de la Ciencia, (4)12 55-63.

Ramos, Y., Virgüez, Y., y Wolff, M. (2018). Insects of forensic importance associated to cadaveric decomposition in a rural area of the andean amazon, Caquetá, Colombia. Acta Amazonica, 48(2), 126-136. doi: /10.1007/978-3-540-71923-6_9.

Ramos, Y., Velasquez, A., y Wolff, M. (2014). Preliminary study of insects associated to indoor body decay in Colombia. Revista Brasileira de Entomologia, 58(4), 326-332. doi:10.1590/s008556262014005000006

Salazar, F., y Donoso, D. (2015). Catálogo de insectos con valor forense en el ecuador. Revista Ecuatoriana de Medicina y Ciencias Biológicas, 36(1),49-59.

Vasconcelos, S., y Araujo, M. C. (2012). Necrophagous species of Diptera and Coleoptera in northeastern Brazil: state of the art and challenges for the Forensic Entomologist. Revista Brasileira de Entomologia, 56(1), 7-14. doi: 10.1590/S0085-56262012005000014.

Villanueva, D., y Seclen, C. (2016). Entomofauna forense y su utilidad en la estimación del intervalo postmortem en cadaveres ahorcados de cerdos (sus seroja l.). Chiclayo, Perú, mayo- octubre 2015. Universidad nacional "Pedro Ruiz Gallo", Perú. 
Zanetti, N., Visciarelli, E. C., y Centeno, N. (2015). Trophic roles of scavenger beetles in relation to decomposition stages and seasons. Revista Brasileira de Entomologia, 59(2), 132-137. doi: 10.1016/j.rbe.2015.03.009.

Para citar el artículo indexado.

Arbeláez, J., \& Narváez, A. (2019). Lista preliminar de dípteros de importancia forense en Guayaquil. Ciencia Digital, 3(1.1), 108-117. https://doi.org/10.33262/cienciadigital.v3i1.1.363

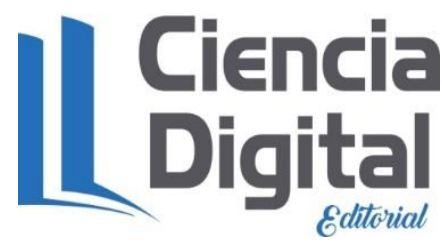

El artículo que se publica es de exclusiva responsabilidad de los autores y no necesariamente reflejan el pensamiento de la Revista Ciencia Digital.

El articulo queda en propiedad de la revista y, por tanto, su publicación parcial y/o total en otro medio tiene que ser autorizado por el director de la Revista Ciencia Digital.
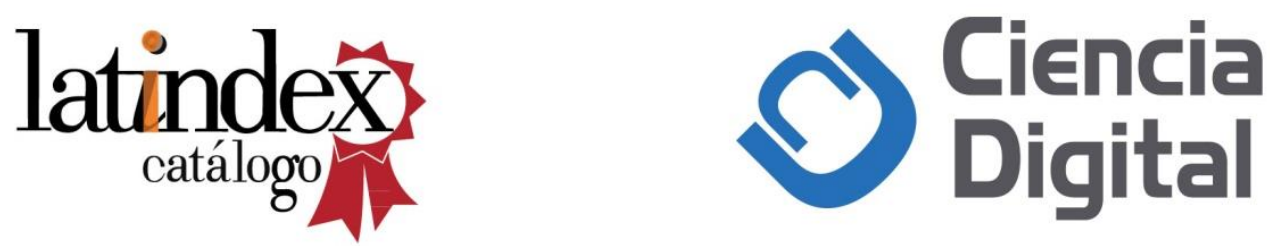